\title{
Three-dimensional Modeling of Intumescent Behavior in Fires
}

\author{
KATHRYN M. BUTLER, HOWARD R. BAUM and TAKASHI KASHIWAGI \\ National Institute of Standards and Technology \\ Gaithersburg, Maryland 20899 USA
}

\begin{abstract}
A method of studying the swelling and thermal behavior of intumescent materials using understanding of the basic physical and chemical processes is described. The material is treated as a highly viscous fluid with properties dependent on temperature. The growth rate, migration, and thermal effects of a large number of bubbles are individually calculated using approximate analytical solutions to mass, momentum, and energy equations, and the collective behavior is obtained as a summation of the individual velocity and temperature fields. The approach and implementation of this model are described and demonstrated.
\end{abstract}

KEY WORDS: Intumescence, Fire retardants, Numerical modeling

\section{INTRODUCTION}

Intumescent materials provide a thermal and physical barrier to slow the transport of heat and the rapid flame spread of fires. On exposure to the heat flux from a fire, the temperature within these materials rises, causing the thermoplastic binder to melt. At a critical temperature, an endothermal chemical reaction releases gases, which form large numbers of small bubbles and result in a swelling of the intumescent layer to many times its original thickness. Solidification through cross-linking provides a thick multicellular char that insulates the protected surface and reduces the amount of material that becomes involved in the fire [1]. Intumescent fire retardants have a significant environmental advantage over halogen-based fire retardants, which are effective but tend to release corrosive, toxic, and potentially obscuring gases in a fire [2].

Careful design of the intumescent system is required to provide effective fire protection. The order and timing of chemical events are critical. For instance, if the thermoplastic binder is too viscous when the gasification reaction begins, the resistance to bubble growth will prohibit the necessary swelling. If the thermoplastic binder is not viscous enough, the bubbles will be large, 
resulting in a fragile char or in a char in which the bursting of bubbles before solidification has prevented swelling. Selection of chemical components for intumescent paints and, recently, of additive systems for commercial polymers [2] has proceeded through empirical methods and been documented primarily in the patent literature. There is as yet only a limited understanding of the physical mechanisms that govern intumescent behavior.

Some understanding of the mechanisms leading to thermal protection has been obtained using one-dimensional models that treat the swelling polymeric material as a single layer with timevarying effective physical parameters $[3,4]$ or as a set of layers consisting of virgin polymer and char separated by a thin pyrolysis zone $[5,6,7]$. These models have identified two mechanisms responsible for slowing the transport of heat. First, the gasification reaction is endothermal, causing energy to be absorbed during the swelling phase. The second mechanism is the reduced thermal conductivity of the final char. Because the rate of swelling must be provided as an input for these models, they are unable to provide insight into the complicated sequence of physical, chemical, and thermal events that characterize intumescent behavior.

The three-dimensional model described in this paper incorporates the fundamental hydrodynamics, chemical reactions, and heat transfer leading to the swelling and thermal protection provided during intumescence. In this model, the intumescent material is represented as a viscous fluid whose viscosity is dependent on temperature. To represent material samples of a size compatible with the cone calorimeter, approximately 10,000 infinitesimal bubble nucleation sites are randomly distributed through the initial geometry. When a heat flux applied to one side of the material raises the temperature at a nucleation site to a critical value for gasification, the bubble begins to grow. Its growth rate, rate of migration, and thermal effects are determined by equations of mass, momentum, and energy solved under local conditions. The collective behavior of the system on a global scale is determined by summing the results of calculations for individual bubbles.

This problem is awkward in the sense that a large number of bubbles must be considered, making accurate solution of the governing equations impossible due to the complex set of boundary conditions at the interface of each bubble and along the sides of the melt. In our approach to this problem, we make approximations that are designed to retain details of the mechanisms yet still enable calculations over a reasonable (one or two day) time period on today's scientific workstations. The eventual goal of this model is to provide a tool for design through improved understanding of the sensitivity of intumescent characteristics to various physical and chemical parameters.

\section{HYDRODYNAMICS SUBMODEL}

The intumescent sample is considered to be an incompressible fluid with viscosity and surface tension dependent on temperature. Initially, the sample takes the shape of a rectangular slab and contains a large number of infinitesimally small bubble nucleation sites randomly distributed throughout the volume. When the temperature at a specific nucleation site reaches the degradation temperature, the bubble begins to grow. We want to know the effects of this bubble growth on the migration of bubbles throughout the melted polymer and on the melt itself. We begin by looking at the velocity field generated by a single expanding bubble. 

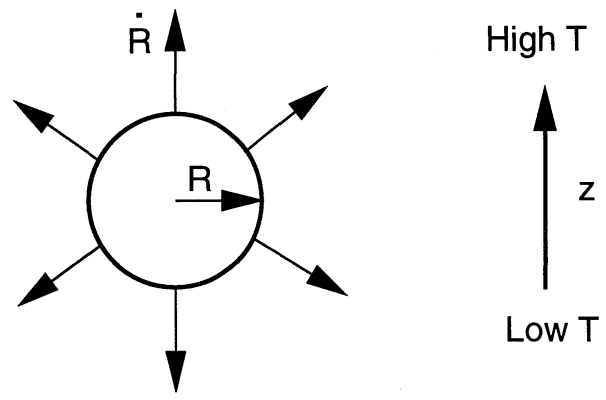

FIGURE 1: Geometry of the expanding bubble in a thermal gradient.

The geometry of a solitary bubble expanding in a uniform temperature gradient is illustrated in Figure 1. The bubble radius is $R$ and its growth rate is $\dot{R}$. If the physical properties of the surrounding melt are constant, then the equations of mass and momentum are solved exactly by radial velocity and pressure fields:

$\mathbf{u}=\hat{\mathbf{e}}_{r} \frac{R^{2} \dot{R}}{r^{2}} \quad ; \quad p=p_{\infty}+\rho\left[\frac{R^{2} \ddot{R}+2 R \dot{R}^{2}}{r}-\frac{R^{4} \dot{R}^{2}}{2 r^{4}}\right]$

where $\mathbf{u}$ is the velocity vector, $\hat{\mathbf{e}}_{r}$ the unit vector in the radial direction, $r$ the distance from the center of the bubble, $p$ the pressure, and $\rho$ the melt density. The presence of a temperature gradient results in spatial variation of the viscosity and surface tension, which, in addition to the buoyancy force, cause the bubble to migrate. For the intumescent problem we can safely assume that the Reynolds number of the translation caused by these forces, $R e=\rho U(2 R) / \mu$, where $U$ is velocity and $\mu$ is melt viscosity, is very small. Assuming also that the translation resulting from these forces is much smaller than the expansion velocity, the bubble will retain a spherical shape throughout its growth.

The effects of gravity and a surface tension gradient on the motion of spheres are well known $[8,9]$, but the force due to the viscosity gradient requires further explanation. For variable viscosity, the Navier-Stokes (NS) equation is

$\rho\left(\frac{\partial \mathbf{u}}{\partial t}+\mathbf{u} \cdot \nabla \mathbf{u}\right)=-\nabla p+\mu \nabla^{2} \mathbf{u}+\nabla \mu \cdot\left[\nabla \mathbf{u}+(\nabla \mathbf{u})^{T}\right]$

where the superscript $T$ indicates the transpose. The gradient of melt viscosity can be written as $\nabla \mu=\nabla T(\partial \mu / \partial T)$, where the variation of viscosity with temperature is estimated by the standard WLF equation for polymer melts [11]. For a bubble whose thermal conductivity is much smaller than that of the surrounding melt, the temperature field is approximated by

$T=T_{0}+G r \cos \theta\left(1+\frac{R^{3}}{2 r^{3}}\right)$, 
where $\mathbf{G}=\hat{\mathbf{e}}_{z} G$ is the local temperature gradient and the angle $\theta$ is measured from the $+z$ axis. Note that a discussion of this temperature field can be found later in this paper in the Heat Transfer Submodel section.

The velocity and pressure fields due to the viscosity gradient, $\mathbf{u}_{1}(r, \theta)$ and $p_{1}(r, \theta)$, are assumed to be perturbations added to the radial fields (1). Subtracting the radial solution from the NS equation and making the assumptions that $R e \ll 1, U \ll \dot{R}$, and $R(\partial \ln \mu / \partial z) \ll 1$ results in the following analytical expressions for velocity and pressure:

$\mathbf{u}_{1}=\frac{R^{2} \dot{R} G}{4 r} \frac{d \ln \mu}{d T}\left[\hat{\mathbf{e}}_{r} \cos \theta\left(\frac{R^{3}}{r^{3}}-1\right)+\hat{\mathbf{e}}_{\theta} \sin \theta\left(\frac{R^{3}}{r^{3}}+\frac{1}{2}\right)\right]$

$p_{1}=\frac{R^{2} \dot{R} G \cos \theta}{r^{2}} \frac{d \mu}{d T}\left(\frac{7}{4}-\frac{R^{3}}{r^{3}}\right)$

We can now determine the drag force on the bubble arising from the pressure, the deviatoric (non-isotropic) normal stress, and the shear stress [10]:

$F=-\int_{S} p \cos \theta d S+\int_{S} 2 \mu \frac{\partial u_{r}}{\partial r} \cos \theta d S-\int_{S} \mu\left(\frac{1}{r} \frac{\partial u_{r}}{\partial \theta}+\frac{\partial u_{\theta}}{\partial r}-\frac{u_{\theta}}{r}\right) \sin \theta d S$.

Substituting expressions (4) and (5) into equation (6), the total drag force is calculated to be

$F=-4 \pi R^{2} \dot{R} \frac{\partial \mu}{\partial T} G$

The terminal velocity is obtained by equating this force to the force on a translating sphere in Stokes flow [8],

$F_{S}=2 \pi R \mu U\left(\frac{2+3 \kappa}{1+\kappa}\right)$

where $\kappa$ is the ratio of sphere viscosity to fluid viscosity. Since even a trace amount of surfaceactive contamination will eliminate convection in the bubble interior, we take $\kappa \rightarrow \infty$, and the translation velocity due to the viscosity gradient becomes

$U=-\frac{2}{3} R \dot{R} \frac{\partial \ln \mu}{\partial T} G$

Velocities due to gravity and to the surface tension gradient are simply added to this quantity.

The flow field in the fluid surrounding a single bubble is given by the sum of the radial expansion field and the flow field for a sphere traveling with velocity $U$. If we can make the assumption that the distance between bubble centers is much larger than their radii, then a reasonable approximation for the total flow field for multiple bubbles is a simple summation of individual flow fields. In this field the bubbles experience forces induced by other bubbles, which tend to push them apart. For the intumescent problem, a condition of no flux across the lower boundary of the sample can be obtained using image bubbles. Figure 2 shows the flow 


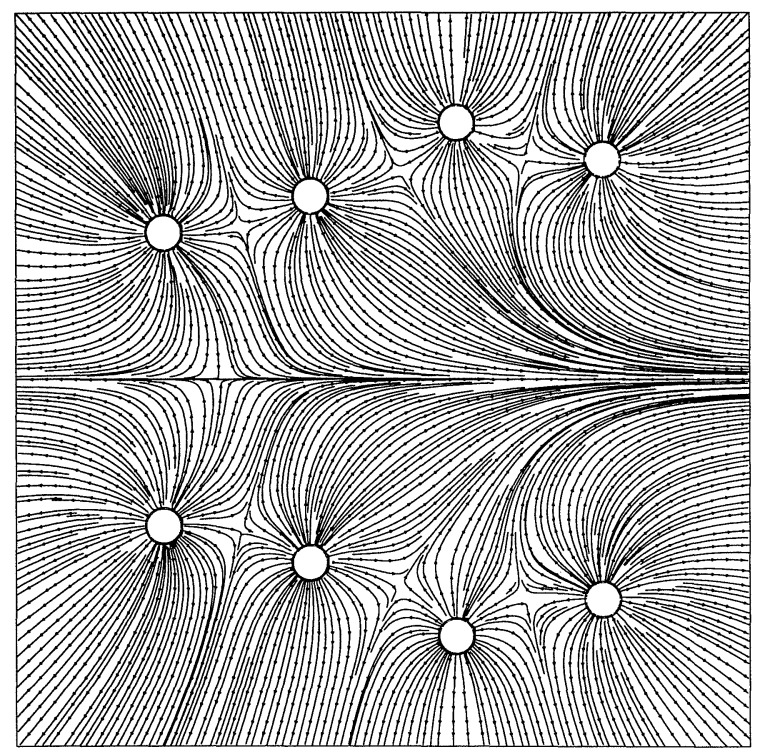

FIGURE 2: Streamlines along plane of symmetry for four bubbles and their images.

field resulting from four coplanar expanding bubbles and their images.

The assumption of large bubble spacing compared to radius holds for early development of intumescence, but breaks down at later times when large bubbles are separated by thin layers of melt. The results for this model will therefore be most accurate during the early stages as the pyrolysis front moves through the material. The swelling of the intumescent surface is determined by the sum of forces from the expanding and migrating bubbles, which move the locations of points on the initially flat upper surface in time. Bursting at the surface is currently prohibited in this model. If a bubble tries to break the upper surface, the location of surface points is adjusted to retain the bubble within the sample. Coalescence is included by replacing two overlapping bubbles by a single bubble whose volume equals the sum of the merged bubbles, centered at the bubbles' center of mass.

\section{GROWTH RATE SUBMODEL}

In the intumescent material, the growth rate of bubbles depends on the chemistry of the decomposition of the blowing agent and on the physical properties of the gas and surrounding melt. When the local temperature has risen sufficiently to trigger decomposition of the blowing agent, the concentration of gas in the polymeric melt begins to rise. Diffusion of gas into nearby nucleation sites provided by impurities in the melt causes the bubbles to begin expanding. Initially, bubble growth is opposed by the surface tension of the melt. In a typical viscous liquid, the growth rate at later stages is controlled by liquid inertia and viscosity, by a com- 


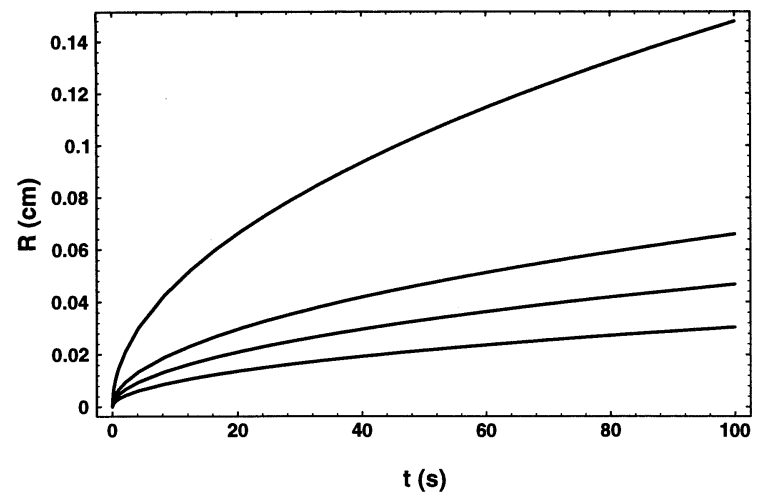

FIGURE 3: Bubble radius vs. time according to the Epstein and Plesset expression. This plot illustrates the effect on growth rate of changes in diffusion coefficient $D$, gas solubility $S^{\prime}$, and ratio of initial supersaturation pressure to minimum critical pressure $P_{0} / P_{c}$. Parameter values for the plots from fastest growing to slowest growing are $\left(D, S^{\prime}, P_{0} / P_{c}\right)=$ $\left(10^{-9}, 1,4\right),\left(10^{-9}, 1,2\right),\left(10^{-10}, 1,4\right),\left(10^{-9}, 0.1,4\right)$ respectively, where $D$ is expressed in units of $\left.\mathrm{m}^{2} / \mathrm{sec}\right)$.

bination of inertial and thermal effects, and, finally, by the transport of heat and mass alone. In the intumescent melt, however, viscous resistance to growth is expected to dominate until crosslinking and char formation causes the melt to solidify. The growth rate is also affected by depletion of the blowing agent, which leads to eventual decline in the gas concentration in the vicinity of the bubble.

As an initial, simplified approach for including bubble growth effects in the three-dimensional model, bubble growth at a specific nucleation site begins when the temperature exceeds a specified critical temperature. The growth rate is given by a simple calculation performed for an oversaturated liquid-gas solution by Epstein and Plesset [12]. The analysis assumes that the dissolved gas concentration satisfies a spherically symmetric diffusion equation. Convection from bubble expansion is neglected, the initial concentration is assumed constant, and the concentration at the bubble interface equals that for a saturated solution. The resulting expression for the change in radius with time is

$\frac{d R}{d t}=D S^{\prime}\left(\frac{P_{0}}{P_{c}}-1\right)\left[\frac{1}{R}+\frac{1}{(\pi D t)^{1 / 2}}\right]$,

where $D$ is the diffusion coefficient, $S^{\prime}$ the gas solubility, $P_{0}$ the initial supersaturation pressure, and $P_{c}$ the minimum critical pressure for bubble inflation. Bubble radius as a function of time for some typical parameter values is shown in Figure 3. Gent and Tompkins [13] have verified that this expression is valid for the growth of bubbles in elastomers, and it has been used to establish that diffusion from small bubbles to large bubbles in a structural foam is unimportant except for very thin bubble walls and low internal bubble pressures. [14] 
A more realistic calculation of bubble growth must include viscous resistance to bubble expansion, reaction chemistry, and response of local physical parameters and chemistry to the temperature. An approach that includes variations in the concentration of dissolved gas in the liquid with radius and time is described by Street et al. [15] and Amon and Denson [16]. These models consider a solitary bubble expanding in a spherical cell containing a specific amount of liquid. Solidification effects are incorporated through variations of viscosity with time. Current efforts to improve the bubble growth calculations in the intumescent model involve modification of this approach to represent the intumescent environment more accurately. In particular, initial increases in gas concentration due to chemical reactions will be related to local temperature through an Arrhenius expression.

\section{HEAT TRANSFER SUBMODEL}

Upon exposure to the heat flux from a fire, the temperature within the intumescent sample rises, triggering gasification reactions at locations progressively farther from the upper surface. In the absence of bubbles, the temperature field is determined by solving a one-dimensional heat transfer equation:

$\frac{\partial T}{\partial t}=\alpha \frac{\partial^{2} T}{\partial z^{2}}$

subject to a heat flux applied at the upper surface. Initially the entire sample is at room temperature. Initial and boundary conditions for this problem are:

$T(z, 0)=T_{0}$

$\frac{\partial T}{\partial z}\left(z_{s}, t\right)=-\frac{\dot{Q}^{\prime \prime}}{k}$

$\lim _{z \rightarrow-\infty} T(z, t)=T_{0}$

Here $\alpha$ is the thermal diffusivity of the melt, $k$ the thermal conductivity of the melt, $\dot{Q}^{\prime \prime}$ the applied heat flux, and $z_{s}$ the location of the upper surface in reference to the lower surface located at $z=0$. Assuming that thermal parameters and the applied heat flux are constant, the solution to this simple problem is

$T(z, t)=\frac{2 \dot{Q}^{\prime \prime}}{k} \sqrt{\frac{\alpha t}{\pi}} \exp \left[\frac{-\left(z_{s}-z\right)^{2}}{4 \alpha t}\right]+\frac{\dot{Q}^{\prime \prime}}{k}\left(z_{s}-z\right) \operatorname{erfc}\left(\frac{z_{s}-z}{2 \sqrt{\alpha t}}\right)$.

The progression of the intumescent front in response to this temperature profile, with the heat transfer effects from the bubbles themselves left out, is illustrated in Figure 4. Note that, although some swelling has occurred, the size and density of bubbles are not sufficient to increase the volume of the sample by a large amount.

Now consider the effects of gas bubbles. We know from the literature that two important factors that slow the transport of heat through intumescent materials are the endothermicity of 

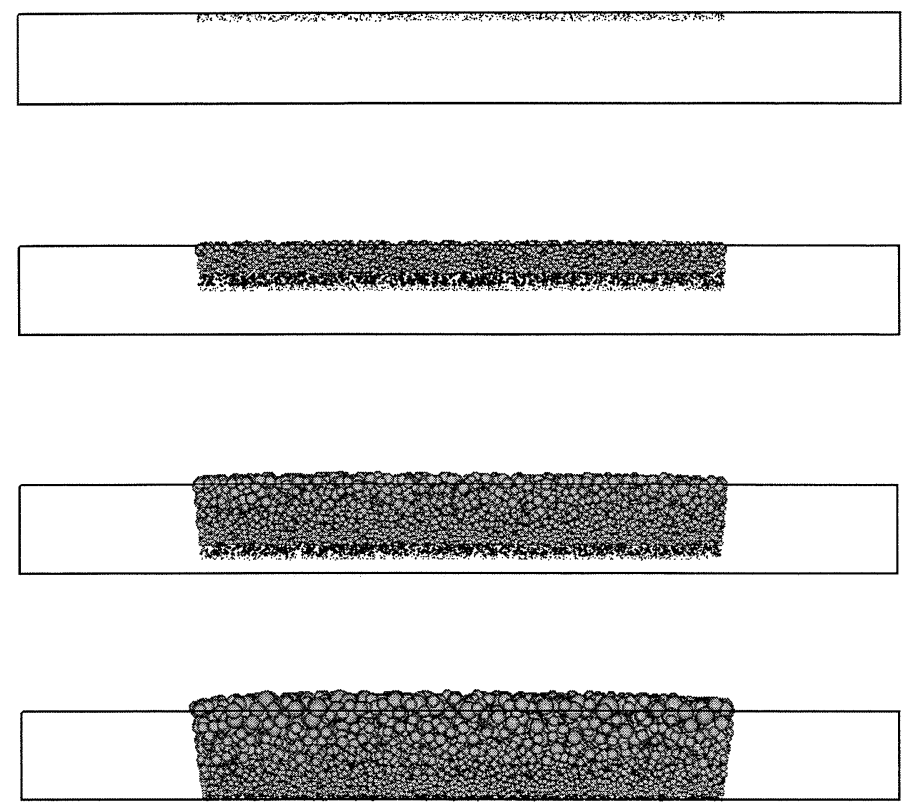

FIGURE 4: Development of bubbles with time as a steady heat flux of $40 \mathrm{~kW} / \mathrm{m}^{2}$ is applied to the upper surface of a rectangular slab with dimensions (in meters) of $0.1 \times 0.1 \times 0.01$. Bubble nucleation sites have been randomly located throughout the central region with dimensions $0.06 \times 0.06 \times 0.01$. The view is taken through the side of the material, which is visualized as transparent, with bubbles shown as opaque.

the gasification reaction and the insulating properties of the final char. The approach we take is to consider the heat transfer in the vicinity of the solitary bubble of Figure 1, with thermal conductivity much lower in the interior than in the exterior, and with an endothermal heat flux due to gasification reactions applied at the bubble surface. In truth, decomposition of the blowing agent occurs throughout the melted polymer, depending on the local temperature. The application of the heat of reaction to the bubble surface rather than distributed through the melt simplifies the problem sufficiently to enable an approximate analytic solution.

The temperature field in the presence of the solitary bubble can be obtained by summing the field in its absence, $T_{1}(z, t)$, as given by (15), with a local field $T_{2}(\mathbf{r}, t)$ that satisfies the appropriate energy equation and enables the total field to match the temperature and heat flux to those of the interior at the bubble wall. A convective term due to bubble expansion is included in the equation for $T_{2}$ :

$\frac{\partial T_{2}}{\partial t}+\frac{R^{2} \dot{R}}{r^{2}} \hat{\mathbf{e}}_{r} \cdot \nabla T_{2}=\alpha \nabla^{2} T_{2}$ 
Since the thermal conductivity of the gas within the bubble is much lower than that of the melt, the total heat flux to the bubble surface must equal the heat flux due to the endothermal reaction $\dot{q}^{\prime \prime}$ to a first approximation. So the boundary condition for $T_{2}$ is

$\hat{\mathbf{e}}_{r} \cdot \nabla T_{2}=-\hat{\mathbf{e}}_{r} \cdot \mathbf{G}-\frac{\dot{q}^{\prime \prime}}{k} \quad$ at $\quad r=R(t)$

where $\mathbf{G}(t)$ is the gradient of $T_{1}$ at the bubble location $z_{b}$ :

$\mathbf{G}=\hat{\mathbf{e}}_{z} \frac{\dot{Q}^{\prime \prime}}{k} \operatorname{erfc}\left(\frac{z_{b}}{2 \sqrt{\alpha t}}\right)$

Assuming that the bubble radius is much smaller than the thickness of the melt, $T_{2}$ must also satisfy $T_{2}(\mathbf{r}, 0)=0$ and $T_{2}(\mathbf{r}, t) \rightarrow 0$ as $r \rightarrow \infty$.

The effects of the time-dependent boundary $r=R(t)$ are absorbed into the energy equation by changing variables from $(r, \theta, t)$ to $(\xi(r, t), \theta, t)$, where $\xi \equiv r / R(t)$. Time $t$ is nondimensionalized by a diffusion time $L^{2} / \alpha$, where $L$ represents a distance of interest in the melt (the distance from the center of the bubble to the melt surface, for example, or the distance to a nearby bubble). The equation to be solved can now be written as

$\frac{R^{2}}{L^{2}} \frac{\partial T_{2}}{\partial \tau}+\frac{R \dot{R}}{\alpha}\left(\frac{1}{\xi^{2}}-\xi\right) \frac{\partial T_{2}}{\partial \xi}=\nabla^{2} T_{2}$

subject to

$\frac{\partial T_{2}}{\partial \xi}=-G R \cos \theta-\frac{\dot{q}^{\prime \prime} R}{k} \quad$ at $\quad \xi=1$

$T_{2}(\xi, 0)=0 \quad ; \quad \lim _{\xi \rightarrow \infty} T_{2}(\xi, \tau)=0$

where $\nabla$ is now in terms of $(\xi, \theta)$ and $\tau$ is the nondimensional time.

Again, for practical reasons we seek a simple analytic solution for the single bubble. First we note that for the intumescent problem, where either bubble radius or growth rate is very small, $R \dot{R} / \alpha \ll 1$ for all times, and we can safely neglect the convective terms. In other words, the time scale for thermal diffusion is much shorter than the time scale for bubble growth. A numerical solution of the diffusion equation gives results that are well approximated by a quasi-steady harmonic solution,

$T_{2}=\frac{G R^{3} z}{2 r^{3}}+\frac{\dot{q}^{\prime \prime} R^{2}}{k r}$

This is a sum of a dipole that represents the thermal conductivity difference and a sink that represents the endothermal reaction. The total temperature fields for a bubble in a uniform positive background temperature gradient showing the separate effects of small thermal conductivity of the bubble and endothermicity are displayed in Figure 5. Here the temperature within the bubble is a function of $\mathrm{z}$ only, and matches the exterior solution at the surface. Note 

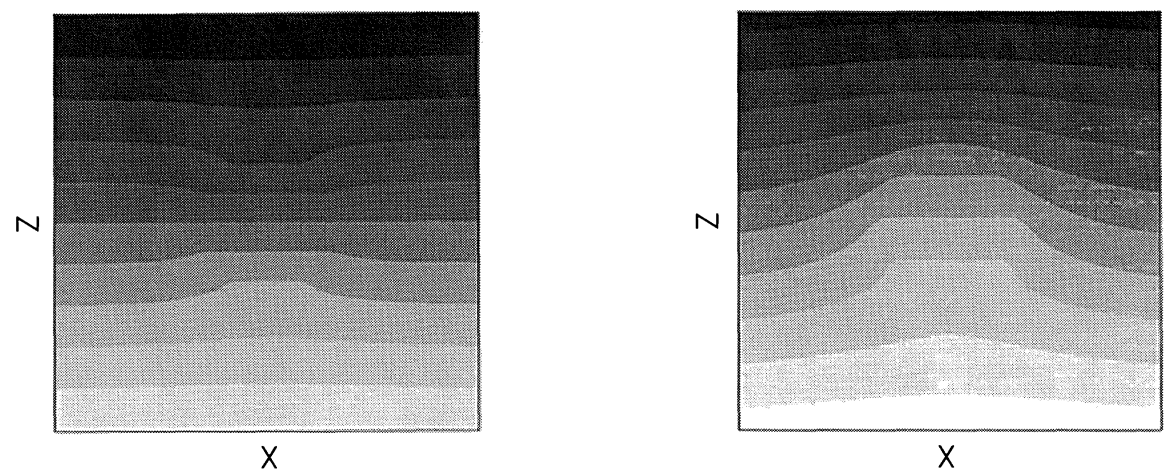

FIGURE 5: Temperature contours for a bubble in a uniform positive background temperature gradient with a low thermal conductivity compared with the surrounding melt (left) and with an endothermal reaction taking place on its surface (right). The bubble surface is located at the discontinuities of the contours, and temperature increases as the shading darkens.

that the temperature below the bubble is reduced in both cases, as we would anticipate.

In this analysis, we have made the assumption that $q$ is steady. The heat flux from the endothermal chemical reaction will, however, vary with local temperature and with the amount of unreacted material remaining. The question of whether this time dependence is adequately represented by setting $\dot{q}^{\prime \prime}$ to $\dot{q}^{\prime \prime}(t)$ in the quasi-steady solution (22) has not yet been addressed.

For multiple bubbles whose separation is much larger than their radii, the total temperature field can be obtained by adding the solution of the energy equation for the melt alone to the sum of fields from individual bubbles responding to local conditions. The rapid distortion of the geometry of the melt by the growing bubbles must be taken into account in these calculations. One approach to this complicated geometry is to introduce a Lagrangian coordinate system, which allows us to solve the heat transfer problem in the original rectangular geometry using finite differences. The evolution in time of a set of expanding bubbles arranged in three planes within the sample is shown in Figure 6. Note that the base of the intumescent material is cooled beneath the bubbles, due to both thermal conductivity effects and to endothermal reactions.

Plans for improving the heat transfer model include a treatment of chemistry that links the production of gases in the bubble growth submodel with the effects of the endothermal heat of reaction on temperature.

\section{THE FULL MODEL}

The submodels of hydrodynamics, bubble growth, and heat transfer are coupled as the model moves forward in time. Initial setup defines the locations of bubble nucleation sites and of node points on a rectangular grid and sets all points at room temperature. At each time step the temperature, temperature gradient, and material properties are determined for each bubble. If 

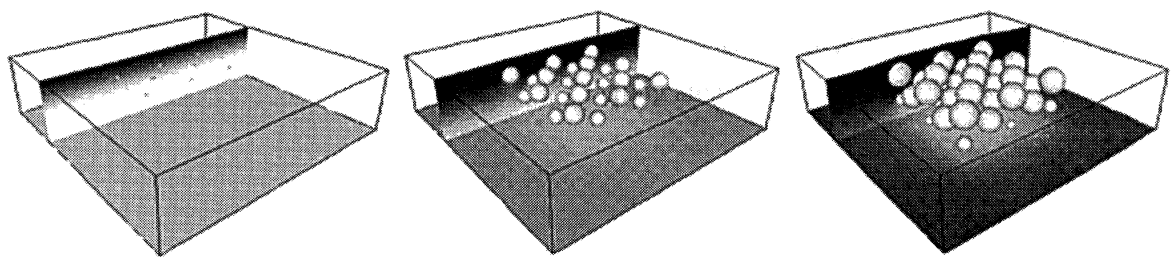

FIGURE 6: Time development of a sample heated from above containing 38 bubbles arranged in three horizontal planes. Bubble thermal conductivity is much smaller than that of the melt, and each bubble acts as a heat sink due to endothermal chemical reactions. Temperature increases as the shading darkens. Note the cooler region (lighter gray) beneath the bubbles.

the critical temperature has been exceeded at a bubble site, its growth rate is set according to radius and time. A Runge-Kutta procedure increments the positions of bubbles and nodes, and the flow field and temperature field are incremented according to the new information.

\section{CONCLUSIONS}

The complex nature of intumescent behavior requires a modeling approach that combines physical and chemical principles for more complete understanding of the mechanisms involved. By combining approximate solutions of mass, momentum, and energy equations on the scale of an individual bubble, the three-dimensional model presented here has demonstrated swelling and cooling of the protected surface, in qualitative agreement with observations. The model includes endothermal chemical reactions, which reduce the temperature at the protected substrate during gasification, and the low thermal conductivity of the bubbles, which reduces heat transport for the final swollen char. The growth rate of bubbles due to diffusion of gases is critical to swelling and heat transport, which are also affected by the migration of bubbles toward regions of higher temperature due to gradients in viscosity and surface tension.

This model is designed for easy incorporation of more detailed submodels as our understanding improves. The addition of phenomena such as char solidification, the effects of viscosity on coalescence and bursting, non-Newtonian fluid effects, and the dependence of gas concentration and heat flux on local temperature and eventual depletion of the blowing agent, is expected to provide increasingly refined quantitative results for comparison with experiment.

\section{ACKNOWLEDGMENTS}

The authors appreciate the partial support of this work by the Federal Aviation Administration under Interagency Agreement DTFA003-92-Z-0018. 


\section{REFERENCES}

[1] Vandersall, H.L., "Intumescent Coating Systems, Their Development and Chemistry," J. Fire \& Flamm., 2, 97-140, 1971.

[2] Camino, G., Costa, L., and Martinasso, G., “ Intumescent Fire-retardant Systems," Poly. Deg. \& Stab., 23, 359-376, 1989.

[3] Anderson, C.E., Ketchum, D.E., and Mountain, W.P, "Thermal Conductivity of Intumescent Chars," J. Fire Sci., 6, 390-410, 1988.

[4] Zverev, V.G., Isakov, G.N., Nesmelov, V.V., and Nazarenko, V.A., "The Heat Transfer Mechanism and Fire Insulation Properties of Some Intumescent Materials," Intern. J. Polymeric Mater., 20, 91-99, 1993.

[5] Cagliostro, D.E., Riccitiello, S.R., Clark, K.J., and Shimizu, A.B., "Intumescent Coating Modeling," J. Fire \& Flam., 6, 205-220, 1975.

[6] Anderson, C.E. and Wauters, D.K., "A Thermodynamic Heat Transfer Model for Intumescent Systems,” Int. J. Engng. Sci., 22, 881-889, 1984.

[7] Buckmaster, J., Anderson, C., and Nachman, A., "A Model for Intumescent Paints," Int. J. Engng. Sci., 24, 263-276, 1986.

[8] Clift, R., Grace, J.R., Weber, M.E., Bubbles, Drops, and Particles Academic Press, New York, 1978, pp. 30-35.

[9] Young, N.O., Goldstein, J.S., and Block, M.J., "The Motion of Bubbles in a Vertical Temperature Gradient,” J. Fluid Mech., 6, 350-356.

[10] Landau, L.D. and Lifshitz, E.M., Fluid Mechanics, Pergamon Press, Oxford, England, 1986, p. 66.

[11] Williams, M.L., Landel, R.F., and Ferry, J.D., "The Temperature Dependence of Relaxation Mechanisms in Amorphous Polymers and Other Glass-forming Liquids," J. Am. Chem. Soc., 77, 3701, 1955.

[12] Epstein, P.S. and Plesset, M.S., "On the Stability of Gas Bubbles in Liquid-Gas Solutions," J. Chem. Phys., 18, 1505-1509, 1950.

[13] Gent, A.N. and Tompkins, D.A., "Nucleation and Growth of Gas Bubbles in Elastomers," J. Appl. Phys., 40, 2520-2525, 1969.

[14] Hobbs, S.Y., "Bubble Growth in Thermoplastic Structural Foams," Polym. Eng. \& Sci., 16, 270-275, 1976.

[15] Street, J.R., Fricke, A.L., and Reiss, L.P., "Dynamics of Phase Growth in Viscous, NonNewtonian Liquids,” Ind. Eng. Chem. Fundam., 10, 54-64, 1971.

[16] Amon, M. and Denson, C.D., "A Study of the Dynamics of Foam Growth: Analysis of the Growth of Closely Spaced Spherical Bubbles," Polym. Eng. \& Sci., 24, 1026-1034, 1984. 